\title{
Kinematic Analysis of a 3-DOF Screening Parallel Mechanism With Control Decoupling
}

\author{
Jun Xie ${ }^{1}$, Feifei Zuo ${ }^{1, *}$, Qizhi Yang ${ }^{2}$, Yuping Li $^{1}$ \\ ${ }^{1}$ School of Mechanical Engineering, Jiangsu University, Zhenjiang, China \\ ${ }^{2}$ School of Agricultural Equipment Engineering, Jiangsu University, Zhenjiang, China \\ *Corresponding Author.
}

\begin{abstract}
In order to raise the low efficiency of traditional screening machine, a new parallel mechanism (PM) with two translation and one rotation is proposed. Analyze the composition of each kinematic chain based on topological characteristics of mechanism The analytical method is utilized to simplify three constraint equations of PM respectively and the correctness is verified by Matlab. On the basis of kinematic analysis, the Jacobian matrix of mechanism is solved, and decoupling characteristics of mechanism input-output control modes are evaluated. The mechanism is coupled between translational input $Y$ and translational output $x$, and three input-output control modes are decoupling. In addition, the singular configuration of PM is also analyzed. The discrete element method was used to simulate material screening, and the influence of DOF on screening efficiency was analyzed by EDEM software and experiment, which result is met with the theoretical analysis.
\end{abstract}

Key words: three degree of freedom (3-DOF), parallel mechanism, singular configuration, control decoupling, screening efficiency.

\section{Introduction}

Based on the principle of material screening, moving sieving movement is conducive to dispersion of materials; Rotating sieving movement can make material fully turn over, prevent the sieving hole from blocking, and speed up screening of feed. In agricultural feed screening operation, the feed screen output trajectory is generally a straight line, circle or ellipse. The single trajectory is bad for rapid dispersion and stratification of feed, which make feed more easy to squeeze each other, block the mesh, and reduce feed screening efficiency. Different from traditional feed screening, the parallel feed screening can make sieving output moving and rotating screening motion at the same time. By combining the advantage of moving and rotating screening motion, the sieving can realize complex spatial motion trajectory, which make effectively improve the quality and efficiency of screening. Moreover, compared with the high-DOF parallel mechanism (DOF>3), the 3-DOF parallel mechanism can realize most complex motion trajectories, with less driving and simple control. Based on the above advantages, 3-DOF parallel mechanism has been widely studied.

In the field of parallel screening machine(SM) research, Shen ${ }^{[1]}$ first proposed a vibrating screen based on parallel mechanism. Xie ${ }^{[2]}$, Qing ${ }^{[3]}$ studied parallel tea SM. Up to now, SM based on PM has not been applied, so the relevant research needs to be carried out. In order to improve the screening efficiency of traditional SM, it is imperative to study multi-degree-of-freedom parallel SM. In terms of mechanism research, $\mathrm{Liu}^{[4]}$ and Dong ${ }^{[5]}$ analyzed the kinematic characteristics of 3-RPR PM. Xu ${ }^{[6]}$ studied (RPall3R)-R+RSS non-parasitic motion PM. In the study of mechanism coupling, Fan ${ }^{[7]}$ made a kinematic analysis of the new $2 \mathrm{R}$ fully decoupled PM. Tian ${ }^{[8]}$ and $\mathrm{Shen}^{[9]}$ studied the coupling degree and decoupling characteristics of 3T1R mechanism.

The main problems associated with the above-mentioned PM:

ISSN: 0010-8189

(C) CONVERTER 2020

www.converter-magazine.info 
Decoupling analysis does not involve the discrimination of coupling degree in control modes, and the decoupling characteristics of input-output control modes are not clear.

The three-degree-of-freedom parallel mechanism proposed in this paper, which three input-output control modes are decoupled under the premise of ensuring the 3-DOF movement of the moving platform.

In order to improve the efficiency of SM, based on the analysis of traditional single DOF SM, a PM with two translation and one rotation is proposed. In the second section, the structure of the three-degree-of-freedom parallel screening machine is introduced in detail. In section 3-4, after the establishment of mathematical model, the forward and inverse kinematics solutions are derived. According to the kinematics solutions, decoupling degree of the control modes of PM is analyzed. In section 5, the singular configuration of the mechanism is analyzed. In section 6, the simulation model of Edem is established, and the screening efficiency of each DOF is analyzed. Finally in section 6 , concluding remarks are presented.

\section{Design of Institution}

According to topology design theory of parallel mechanism based on position and orientation characteristic, the 2T1R parallel mechanism consists of moving platform 1, static platform 0 and single opened chain I, II, III, the topological structure of parallel mechanism is PRR+2-PRRU.

As shown in Fig 1, the structure of single open chain $I\left(P_{1} \square R_{1} \square R_{2}-U_{1}\right)$ is equivalent to $\left(P_{1} \square R_{1} \square R_{2} \square R_{11} \perp R_{12}\right)$, and the structure of single open chain II $\left(\mathrm{P}_{2} \square R_{3} \square R_{4}-\mathrm{U}_{2}\right)$ is equivalent to $\left(\mathrm{P}_{2} \square \mathrm{R}_{3} \square \mathrm{R}_{4} \square \mathrm{R}_{21} \perp \mathrm{R}_{22}\right)$, the structure of single open chain III is $\left(\mathrm{P}_{3} \square \mathrm{R}_{5} \square \mathrm{R}_{6}\right)$. Among them, the single-open chain I makes screen output a translational movement along $\mathrm{x}$-axis, the single-open chain II makes screen output a rotational movement around $\mathrm{y}$-axis, and the single-open chain III makes screen output a translational movement along y-axis.

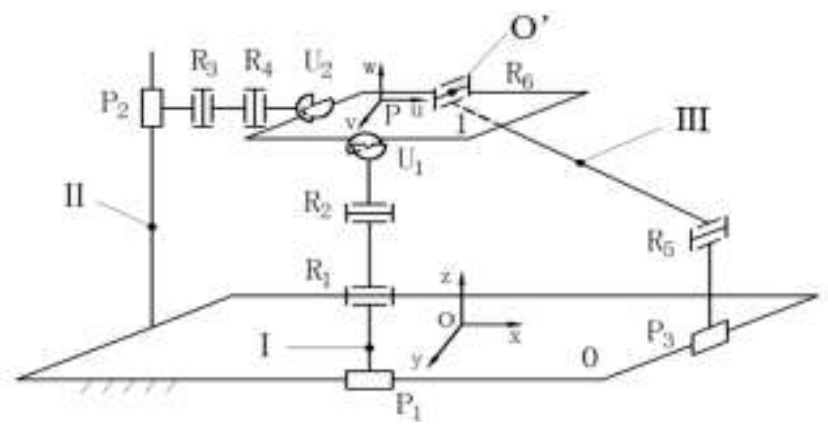

Fig 1: Schematic diagram of 2T1R parallel mechanism PRR+2-PRRU

\section{Kinematic Analysis}

As shown in Fig 2, the distance between fixed platform 0 and screen 1 is $L_{0}$, the coordinate systems $O-\{x y z\}$ and $\mathrm{P}-\{\mathrm{uvw}\}$ are established on fixed platform and screen in turn. The dimensions of each component in this parallel mechanism are: the static platform 0 is $2 l_{l}$ in length and $2 l_{2}$ in width, the screen 1 is $2 l_{3}$ in length and $2 l_{4}$ in widthg, $B_{3} C_{3}=l_{5}, A_{3} B_{3}=l_{6}, A_{2} B_{2}=l_{7}, B_{2} C_{2}=l_{8}$ and $C_{2} D_{2}=l_{9}$.

ISSN: 0010-8189 


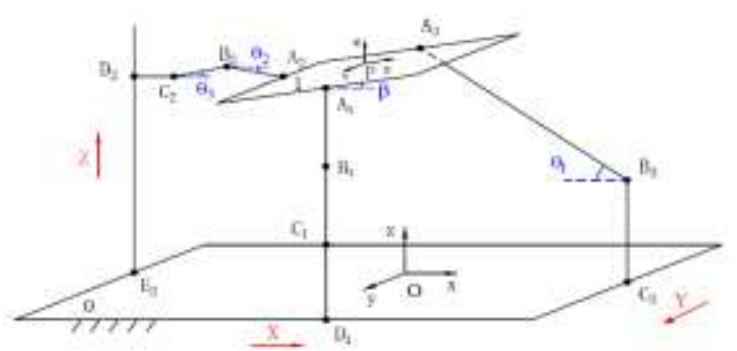

Fig 2: Kinematics model of closed loop

\subsection{Forward kinematic}

It can be seen from Fig 2 that mechanism makes screen output $(x, y, \beta)$ move under the action of input motion $(X, Y, Z)$, the forward kinematics ${ }^{[10]}$ of mechanism is the process of solving $(x, y, \beta)$ from $(X, Y, Z)$, and the inverse kinematics of mechanism is the process of solving $(X, Y, Z)$ from $(x, y, \beta)$.

(1) Calculation process of $x$ and $y$

As shown in Fig 1, during the movement of PM, the displacement of prismatic pair $\mathrm{P}_{1}$ is the displacement of moving platform along the $\mathrm{u}$ axis, then:

$$
x=X
$$

As shown in Fig 2, When the displacement of driving pair $\mathrm{P}_{2}$ is $\mathrm{Z}$, the displacement of driving pair $\mathrm{P}_{3}$ is $\mathrm{Y}$, and the displacement of driving pair $\mathrm{P}_{1}$ is $\mathrm{X}$, the included angle between moving platform and $x$-axis is $\beta$, the included angle between linkage $A_{3} B_{3}$ and $x$-axis is $\theta_{1}$, the included angle between linkage $A_{2} B_{2}$ and $x$-axis is $\theta_{2}$, the included angle between linkage $B_{2} C_{2}$ and $x$-axis is $\theta_{3}$. As shown in Fig $2, C_{3}=\left(l_{1}, Y, 0\right), B_{3}=\left(l_{1}, Y, l_{5}\right)$ in fixed coordinate system $\mathrm{O}$ and $A_{3}=\left(0,-l_{4}, 0\right)$ in moving coordinate system P. Point $A_{3}$ is transformed to fixed coordinate system $\mathrm{O}$ :

$$
A_{3}^{\prime}=T \cdot A_{3}+O=\left[\begin{array}{c}
x \\
y-l_{4} \\
L_{0}
\end{array}\right]
$$

where: rotation matrix $T=\left[\begin{array}{ccc}\cos \beta & 0 & \sin \beta \\ 0 & 1 & 0 \\ -\sin \beta & 0 & \cos \beta\end{array}\right]$, translation matrix $O=\left[\begin{array}{c}x \\ y \\ L_{0}\end{array}\right]$, according to the limitation of linkage length $A_{3} B_{3}=l_{6}$, namely:

$$
\left(x-l_{1}\right)^{2}+\left(y-Y-l_{4}\right)^{2}+\left(L_{0}-l_{5}\right)^{2}=l_{6}^{2}
$$

Furthermore, formula(3) can be sorted into:

ISSN: 0010-8189

C CONVERTER 2020 


$$
y= \pm \sqrt{l_{6}^{2}-\left(L_{0}-l_{5}\right)^{2}-\left(x-l_{1}\right)^{2}}+\left(Y+l_{4}\right)
$$

(2) Calculation process of $\beta$

According to the closed loop consisted by kinematic chain II and III, the following equations were established:

$$
\left\{\begin{array}{l}
l_{5}+l_{6} \sin \theta_{1}+l_{3} \sin \beta=L_{0}+Z \\
l_{6} \cos \theta_{1}+l_{3} \cos \beta+l_{7} \cos \theta_{2}+l_{8} \cos \theta_{3}+l_{9}=2 l_{1} \\
l_{9}+l_{8} \cos \theta_{3}+l_{7} \cos \theta_{2}+l_{3} \cos \beta=l_{1}+X
\end{array}\right.
$$

Equation (7) can be arranged as $l_{8} \cos \theta_{3}+l_{7} \cos \theta_{2}=l_{1}+X-l_{9}-l_{3} \cos \beta$, Substitute into equation (6), and the result can be obtained:

$$
\left\{\begin{array}{l}
l_{6} \sin \theta_{1}=L_{0}+Z-l_{5}-l_{3} \sin \beta \\
l_{6} \cos \theta_{1}=l_{1}-X
\end{array}\right.
$$

Square and add the two equations of equation (8):

$$
l_{3}^{2} \sin ^{2} \beta-2 l_{3}\left(L_{0}+Z-l_{5}\right) \sin \beta+\left(l_{1}-X\right)^{2}-l_{6}^{2}+\left(L_{0}+Z-l_{5}\right)^{2}=0
$$

Further, equation (9) was obtained:

$$
A \sin ^{2} \beta+B \sin \beta+C=0
$$

Where:

$$
\left\{\begin{array}{l}
A=l_{3}^{2} \\
B=-2 l_{3}\left(L_{0}+Z-l_{5}\right) \\
C=\left(l_{1}-X\right)^{2}-l_{6}^{2}+\left(L_{0}+Z-l_{5}\right)^{2}
\end{array}\right.
$$

Arranged equation (10):

$$
U=\sin \beta=\frac{-B \pm \sqrt{B^{2}-4 A C}}{2 A}
$$

That is:

$$
\beta=\arcsin U
$$

3.2 Inverse kinematic

(1) Calculation $X$ and $Y$

ISSN: 0010-8189

C CONVERTER 2020 


$$
\left\{\begin{array}{l}
X=x \\
Y=\mp \sqrt{l_{6}^{2}-\left(L_{0}-l_{5}\right)^{2}-\left(x-l_{1}\right)^{2}}+\left(y-l_{4}\right)
\end{array}\right.
$$

\section{(2) Calculation of $Z$}

According to equation (9), it can be known that:

$$
\left(L_{0}+Z-l_{5}\right)^{2}+d\left(L_{0}+Z-l_{5}\right)+e=0
$$

Where:

$$
\left\{\begin{array}{l}
d=-2 l_{3} \sin \beta \\
e=\left(l_{1}-X\right)^{2}-l_{6}^{2}+l_{3}^{2} \sin ^{2} \beta
\end{array}\right.
$$

Solution of equation (14) was obtained as follows:

$$
\left(L_{0}+Z-l_{5}\right)=\frac{-d \pm \sqrt{d^{2}-4 e}}{2}=u
$$

That is:

$$
Z=u+l_{5}-L_{0}
$$

\section{Decoupling Analysis}

\subsection{Coupling type judgment}

Decoupling ${ }^{[12]}$ means that the motion of the PM in certain direction only depends on the action of driving unit in one direction, while motion in the other direction does not affect the motion in that direction. With the increase of motion branch chain, the coupling of mechanism was strengthened, which further affected the control of mechanism and reduced kinematic accuracy. Therefore, decoupling analysis is necessary.

Assuming the output velocity vector $\dot{p}=(\dot{x}, \dot{y}, \dot{\beta})^{T}$ and the input velocity vector $\dot{q}=(\dot{X}, \dot{Y}, \dot{Z})^{T}$. By taking first derivative of equation (1), (3) and (9) by time, the velocity formulas of the three driving sliders were obtained:

$$
\left\{\begin{array}{l}
\dot{x}=\dot{X} \\
\left(x-l_{1}\right) \dot{x}+\left(y-Y-l_{4}\right) \dot{y}=\left(y-Y-l_{4}\right) \dot{Y} \\
{\left[l_{3}^{2} \sin \beta \cos \beta-l_{3}\left(L_{0}+Z-l_{5}\right) \cos \beta\right] \dot{\beta}=\left(l_{1}-X\right) \dot{X}+\left[l_{3} \sin \beta-\left(L_{0}+Z-l_{5}\right)\right] \dot{Z}}
\end{array}\right.
$$

According to equation (17), based on the Jacobian matrix, the following formula was obtained:

$$
J_{P} \dot{p}=J_{q} \dot{q}
$$

Where $J_{p}$ and $J_{q}$ are the forward and inverse Jacobian matrices of PM respectively.

ISSN: 0010-8189

(C) CONVERTER 2020 
Volume 2021, No. 2

$$
\begin{gathered}
J_{p}=\left[\begin{array}{ccc}
1 & 0 & 0 \\
x-l_{1} & y-Y-l_{4} & 0 \\
0 & 0 & l_{3}^{2} \sin \beta \cos \beta-l_{3}\left(L_{0}+Z-l_{5}\right) \cos \beta
\end{array}\right] \\
J_{q}=\left[\begin{array}{ccc}
1 & 0 & 0 \\
0 & y-Y-l_{4} & 0 \\
l_{1}-X & 0 & l_{3} \sin \beta-\left(L_{0}+Z-l_{5}\right)
\end{array}\right]
\end{gathered}
$$

According to the coupling characteristics, Gogu ${ }^{[11]}$ divides PM into four categories:

(1) If the Jacobian matrix J of PM is a diagonal matrix, and the diagonal elements are same, called the completely homogeneous PM

(2) If the Jacobian matrix J of PM is a diagonal matrix, but the diagonal elements are different, called the decoupling $\mathrm{PM}$

(3) If the Jacobian matrix J of PM is a triangular matrix called the decoupling PM

(4) Except the above three categories, called the coupling PM

With the moving platform outputs $(x, y, \beta)$, there are 7 input-output control modes:

(1) $X-x$, that is, keep the input $(Y, Z)$ unchanged, change the input $X$, and study the corresponding relationship between the input $X$ and the output $x$

(2) $Y-y$, that is, keep the input $(X, Z)$ unchanged, change the input $Y$, and study the corresponding relationship between the input $Y$ and the output $y$.

(3) $Z-\beta$, that is, keep the input $(X, Y)$ unchanged, change the input $Z$, and study the corresponding relationship between the input $(X, Y)$ and the output $\beta$.

(4) $(X, Y)-(x, y)$, that is, keep the input $Z$ unchanged, change the input $(X, Y)$, and study the corresponding relationship between input $(X, Y)$ and output $(x, y)$.

(5) $(X, Z)-(x, \beta)$, that is, keep the input $Y$ unchanged, change the input $(X, Z)$, and study the corresponding relationship between input $(X, Z)$ and output $(x, \beta)$.

(6) $(Y, Z)-(y, \beta)$, that is, keep the input $X$ unchanged, change the input $(Y, Z)$, and study the corresponding relationship between input $(Y, Z)$ and output $(y, \beta)$

(1) $(X, Y, Z)-(x, y, \beta)$, that is, change the input $(X, Y, Z)$ at the same time, and study the corresponding relationship between input $(X, Y, Z)$ and output $(x, y, \beta)$.

ISSN: 0010-8189

C CONVERTER 2020 


\subsection{Control modes decoupling analysis}

According to $J_{p}$ in equation (18), the columns of the forward Jacobian matrix $J_{p}$ reflect the mapping of input-output parameters. In order to further study the decoupling characteristics of mechanism control modes, based on the correlation of Jacobian matrix columns ${ }^{[12]}$, it can be known that:

$$
J_{p}^{\mathrm{T}}(;, i) J_{p}(;, j)=0,(i \neq j ; i=1,2,3 ; j=1,2,3)
$$

Formula (19) shows that the column $i$ is linearly independent of column $j$, which means the input-output control modes represented by column $i$ and $j$ are decoupling.

$$
J_{p}^{\mathrm{T}}(;, i) J_{p}(;, j) \neq 0,(i \neq j ; i=1,2,3 ; j=1,2,3)
$$

Formula (20) shows that the $i$ th column vector is linearly dependent of the $j$ th column vector, which means the input-output control modes represented by $i$ th and $j$ th column vector have coupling.

$$
G=J_{p}^{\mathrm{T}} J_{p}
$$

If $\mathrm{G}$ is a diagonal matrix, the input-output control modes of mechanism is completely decoupled. Otherwise, the input-output control modes is coupled and corresponding input-output control modes is correlated. It can be seen from equation (21):

$$
\begin{aligned}
G & =J_{p}^{T} J_{p} \\
& =\left[\begin{array}{ccc}
1+\left(x-l_{1}\right)^{2} & \left(x-l_{1}\right) \cdot\left(y-Y-l_{4}\right) & 0 \\
\left(x-l_{1}\right) \cdot\left(y-Y-l_{4}\right) & \left(y-Y-l_{4}\right)^{2} & 0 \\
0 & 0 & {\left[l_{3}^{2} \sin \beta \cos \beta-l_{3}\left(L_{0}+Z-l_{5}\right) \cos \beta\right]^{2}}
\end{array}\right]
\end{aligned}
$$

Where $J_{p}^{\mathrm{T}}(;, 1) J_{p}(;, 2)=J_{p}^{\mathrm{T}}(;, 2) J_{p}(;, 1) \neq 0$, from equation $(20)$, there is coupling between translational input $Y$ and translational output $x$. As translational input $Y$ changes, translational output $x$ and $Y$ change simultaneously, that is, the control modes $Y-y,(X, Y)-(x, y),(Y, Z)-(y, \beta)$ and $(X, Y, Z)-(x, y, \beta)$ are coupled. Similarly, the control modes $X-x, Z-\beta$ and $(X, Z)-(x, \beta)$ are decoupled.

\section{Singular Configuration Analysis}

Singular configuration means that when movement of mechanism reaches a specific configuration, the actual number of DOF changes, and mechanism either loses its original DOF and becomes rigid, or gains additional DOF and loses control. Both of these two situations will cause mechanism to be singular, reduce reliability of mechanism's movement. Singular configuration analysis should be carried out in design of PM to avoid area where singular configuration is located, and to reduce the influence of singular configuration on movement of mechanism. Based on the Jacobian matrix, the solutions which make determinant of Jacobian square matrix singular were got. According to whether the Jacobian matrix is singular, the singular configuration of mechanism is divided into input singularity, output singularity and mixed singularity.

5.1 Input singularity analysis

ISSN: 0010-8189

(C) CONVERTER 2020 
When $\operatorname{det}\left(J_{q}\right)=0$ and $\operatorname{det}\left(J_{p}\right) \neq 0$, a member of the mechanism loses its ability to move due to reaching the working boundary. At this time, the mechanism will have input singularity, namely:

$$
\left\{\begin{array}{l}
\left|J_{p}\right|=\left|\begin{array}{ccc}
1 & 0 & 0 \\
x-l_{1} & y-Y-l_{4} & 0 \\
0 & 0 & l_{3}^{2} \sin \beta \cos \beta-l_{3}\left(L_{0}+Z-l_{5}\right) \cos \beta
\end{array}\right| \neq 0 \\
\left|J_{q}\right|=\left|\begin{array}{ccc}
1 & 0 & 0 \\
0 & y-Y-l_{4} & 0 \\
l_{1}-X & 0 & l_{3} \sin \beta-\left(L_{0}+Z-l_{5}\right)
\end{array}\right|=0
\end{array}\right.
$$

(1)When $\left\{\begin{array}{l}l_{3} \sin \beta-\left(L_{0}+Z-l_{5}\right)=0 \\ l_{1}-X=0\end{array}\right.$, it can be seen from equation (8):

$$
\left\{\begin{array}{l}
l_{6} \sin \theta_{R_{1}}=0 \\
l_{6} \cos \theta_{R_{1}}=0
\end{array}\right.
$$

At this time, $\theta_{R_{1}}$ has no solution.

(2)When $y-Y-l_{4}=0, \operatorname{det}\left(J_{q}\right)=\operatorname{det}\left(J_{p}\right)=0$, it does not meet the above judgment conditions.

(3)When $l_{3} \sin \beta-\left(L_{0}+Z-l_{5}\right)=0$, $\operatorname{det}\left(J_{q}\right)=\operatorname{det}\left(J_{p}\right)=0$, it does not meet the above judgment conditions.

\subsection{Output singularity analysis}

When $\operatorname{det}\left(J_{p}\right)=0$ and $\operatorname{det}\left(J_{q}\right) \neq 0$, the moving platform of mechanism can still produce local DOF without input. At this time, the mechanism will have output singularity, namely:

$$
\left\{\begin{array}{l}
\left|J_{p}\right|=\left|\begin{array}{ccc}
1 & 0 & 0 \\
x-l_{1} & y-Y-l_{4} & 0 \\
0 & 0 & l_{3}^{2} \sin \beta \cos \beta-l_{3}\left(L_{0}+Z-l_{5}\right) \cos \beta
\end{array}\right|=0 \\
\left|J_{q}\right|=\left|\begin{array}{ccc}
1 & 0 & 0 \\
0 & y-Y-l_{4} & 0 \\
l_{1}-X & 0 & l_{3} \sin \beta-\left(L_{0}+Z-l_{5}\right)
\end{array}\right| \neq 0
\end{array}\right.
$$

(1)For $l_{3}^{2} \sin \beta \cos \beta-l_{3}\left(L_{0}+Z-l_{5}\right) \cos \beta=0$, when $\beta \neq 90^{\circ}$, the above formula can be simplified to:

$$
l_{3} \sin \beta-\left(L_{0}+Z-l_{5}\right)=0
$$

At this time, $\operatorname{det}\left(J_{q}\right)=\operatorname{det}\left(J_{p}\right)=0$, the judgment conditions are not met.

ISSN: 0010-8189

(C) CONVERTER 2020 
When $\beta=90^{\circ}$, the above formula $\mathrm{Z}$ has infinite solutions, and the solution set is meaningless.

(2)When $\left\{\begin{array}{l}x-l_{1}=0 \\ y-Y-l_{4}=0\end{array}, \operatorname{det}\left(J_{q}\right)=\operatorname{det}\left(J_{p}\right)=0\right.$, the judgment condition is not met.

(3)When $y-Y-l_{4}=0, \operatorname{det}\left(J_{q}\right)=\operatorname{det}\left(J_{p}\right)=0$ the judgment condition is not met.

5.3 Mixed singularity analysis

When $\operatorname{det}\left(J_{p}\right)=\operatorname{det}\left(J_{q}\right)=0$, the mechanism will have mixed singularity:

$$
\left\{\begin{array}{l}
\left|J_{p}\right|=\left|\begin{array}{ccc}
1 & 0 & 0 \\
x-l_{1} & y-Y-l_{4} & 0 \\
0 & 0 & l_{3}^{2} \sin \beta \cos \beta-l_{3}\left(L_{0}+Z-l_{5}\right) \cos \beta
\end{array}\right|=0 \\
\left|J_{q}\right|=\left|\begin{array}{ccc}
1 & 0 & 0 \\
0 & y-Y-l_{4} & 0 \\
l_{1}-X & 0 & l_{3} \sin \beta-\left(L_{0}+Z-l_{5}\right)
\end{array}\right|=0
\end{array}\right.
$$

(1)When $l_{3} \sin \beta-\left(L_{0}+Z-l_{5}\right)=0$, it can be seen from Equation (8):

$$
l_{6} \sin \theta_{R_{1}}=0
$$

That is, if $\theta_{R_{1}}=0^{\circ}$ or $\theta_{R_{1}}=180^{\circ}$, connecting rod $l_{6}$ is perpendicular to the length of moving platform, the mechanism will be singular, and the singular configuration is shown in Fig 3 and Fig 4 .

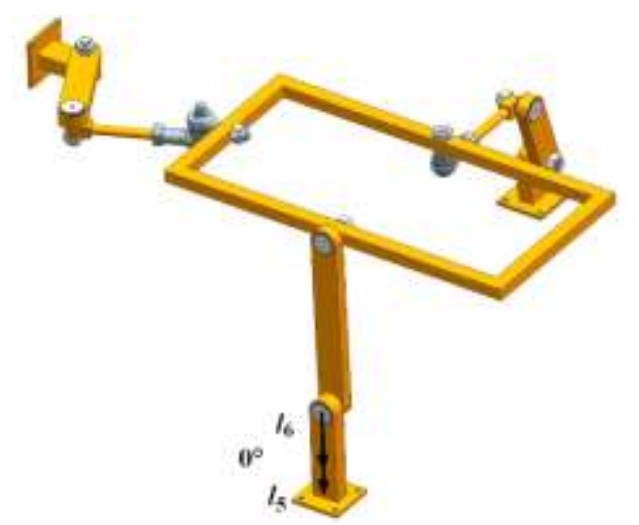

Fig 3: Singular configuration of mechanism with $\theta_{R_{1}}=0^{\circ}$

ISSN: 0010-8189

C CONVERTER 2020 


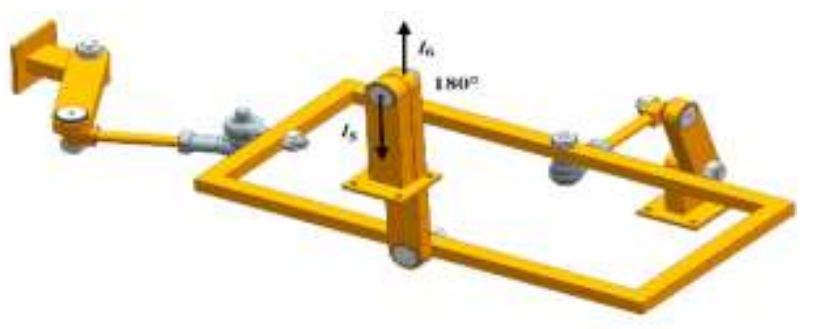

Fig 4: Singular configuration of mechanism with $\theta_{R_{1}}=180^{\circ}$

(2)When $y-Y-l_{4}=0$, from equation (3) we can see:

$$
\left(x-l_{1}\right)^{2}+\left(L_{0}-l_{5}\right)^{2}=l_{6}^{2}
$$

That is, when $x=\sqrt{l_{6}^{2}-\left(L_{0}-l_{5}\right)^{2}}+l_{1}$, the mechanism will have mixed singularity.

\section{Discrete Element Simulation and Experiment}

Compared with traditional sieving machine, the 3-DOF PM can realize material particles translate along $\mathrm{x}, \mathrm{y}$ axis and rotate around y axis. As an analytical method of granular materials, discrete element method is widely used in various fields, in which material particles are regarded as a series of discrete independent motion units, and the objects to be analyzed are regarded as a collection of discrete particles. In order to verify the influence of $2 \mathrm{~T} 1 \mathrm{R}$ screening movement on sieving efficiency, discrete element method was used to simulate the screening process by given screen amplitude and frequency.

As shown in Fig 5, the model of sieving machine was established by Ug software, which is composed of three parts: feeding inlet, screening part and closed shell. The aperture of screening mesh is $6 \mathrm{~mm}$, whose size is $240 \mathrm{~mm} \times 90 \mathrm{~mm}$. After the model was imported into Edem, particle factory was set up at the feeding inlet. The total number of particles is 1500 and the particle size is $6 \mathrm{~mm}$, which produced a normal distribution with mean of 1 and standard deviation of 0.5 .

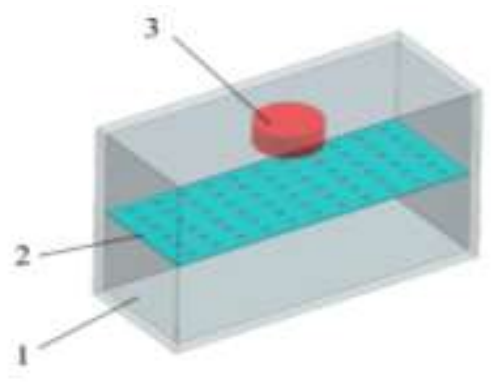

1. closed shell; 2. screening part; 3 . feeding inlet

Fig 5: Sifting machine model

As shown in Table 1 and Table 2 material parameters of material particles and screening body were set respectively.

Table 1 Material parameters of material particles

ISSN: 0010-8189

(C) CONVERTER 2020 
CONVERTER MAGAZINE

Volume 2021, No. 2

\begin{tabular}{|c|c|c|c|c|}
\hline Parameters & Radius/mm & $\begin{array}{c}\text { Poisson's } \\
\text { ratio }\end{array}$ & Solids Density $/ \mathrm{kg} \cdot \mathrm{m}^{-3}$ & $\begin{array}{c}\text { Shear } \\
\text { Modulus/pa }\end{array}$ \\
\hline Numerical value & 6 & 0.3 & 1053 & $1 \mathrm{e}+07$ \\
\hline
\end{tabular}

Table 2 Material parameters of steel

\begin{tabular}{|c|c|c|c|}
\hline Parameters & Poisson's ratio & Solids Density $/ \mathrm{kg} \cdot \mathrm{m}^{-3}$ & Shear Modulus $/ \mathrm{pa}$ \\
\hline Numerical value & 0.3 & 7850 & $1 \mathrm{e}+10$ \\
\hline
\end{tabular}

As shown in Table 3 the interaction parameters between material particles and between material particles and the screening body were set.

Table 3 Material parameters of each materials

\begin{tabular}{|c|c|c|c|}
\hline Parameters & Poisson's ratio & Solids Density $/ \mathrm{kg} \cdot \mathrm{m}^{-3}$ & Shear Modulus/pa \\
\hline Numerical value & 0.3 & 7850 & $1 \mathrm{e}+10$ \\
\hline
\end{tabular}

Set rayleigh time step to $25 \%$, the number of particles to 3000 , simulation time to $5 \mathrm{~s}$, and count the number of sieved particles. Statistical data is divided by total number of particles to obtain particle sieving efficiency data, after simulation ends, which were imported into the Origin software to draw sieving efficiency curve of sieving movement.

Compared with traditional single DOF feed sieving machine, the 3-DOF feed sieving machine based on parallel mechanism can realize screening movement of feed particles along the $\mathrm{x}, \mathrm{y}$ axis and around $\mathrm{y}$ axis by screen. As shown in Fig 6, screening efficiency of 2T1R screening movement is $90.38 \%$, while the screening efficiency of single DOF moving screening movement along the $\mathrm{x}$-direction, along the $\mathrm{y}$-direction, and around the $\mathrm{y}$-direction is $49.4 \%, 42.67 \%$ and $53.47 \%$ respectively. The comparison shows that screening efficiency of 3-DOF screening movement is higher than three kinds of single DOF screening movement.

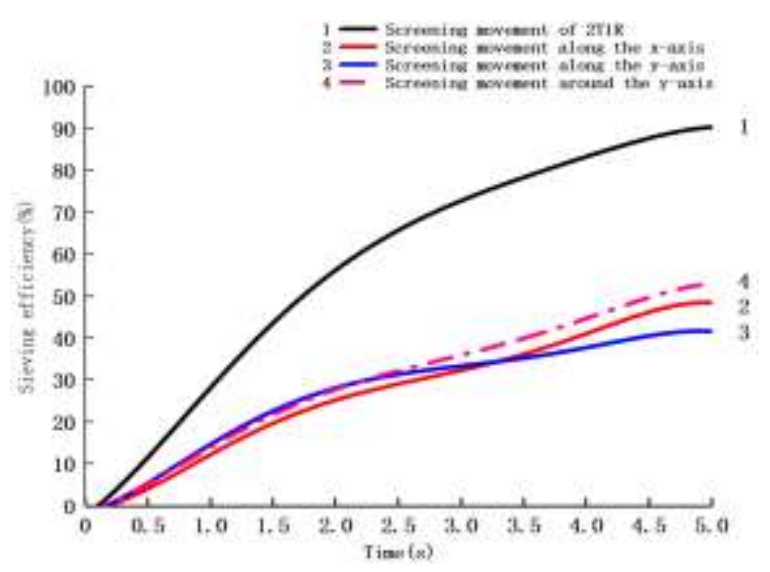

Fig 6: Sieving efficiency curves of 3-DOF and single DOF screen movement

Set up a screening test bench as shown in Fig 6, and use equation(22) to calculate the screening efficiency.

$$
\eta=\frac{W_{1}}{W} \times 100 \%
$$

Where:

ISSN: 0010-8189

(C) CONVERTER 2020 
$W \longrightarrow$ total feed weight

$W_{1} \longrightarrow$ weight of sieved feed

Taking DOF of screen as independent variable and keeping other variables unchanged, the four screening motions in Edem were tested in turn. The test results were shown in the following table:

Table 4 Screening test results

\begin{tabular}{|c|c|c|}
\hline Screen freedom & Screen movement form & $\begin{array}{c}\text { Screening } \\
\text { efficiency }\end{array}$ \\
\hline 1 & single DOF moving screening movement along the x-direction & $63.8 \%$ \\
\hline 1 & single DOF moving screening movement along the y-direction & $62.3 \%$ \\
\hline 1 & single DOF moving screening movement around the y-direction & $69.1 \%$ \\
\hline 3 & 2T1R screening movement & $88.7 \%$ \\
\hline
\end{tabular}

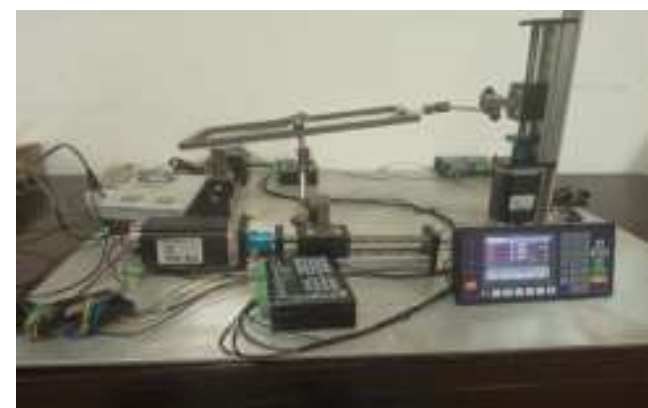

Fig 7: 3-DOF parallel feed sieving machine test equipment

Test results are consistent with the Edem simulation analysis, screening efficiency of 3-DOF screening movement is much higher than three kinds of single DOF screening movement. 3-DOF screening movement can significantly improve the screening efficiency of feed.

\section{Conclusion}

In order to improve the efficiency of traditional SM, a novel type of 2T1R PM with low coupling degree is proposed, which can realize translation along the $\mathrm{x}, \mathrm{y}$ axis and rotation about the $\mathrm{y}$ axis. According to Jacobian matrix, the decoupling type of mechanism was obtained. Moreover, velocity and acceleration were analyzed based on forward and inverse kinematic. Variations of screening efficiency with different DOF were also investigated. Results show that the PM only had coupling between the translational input $\mathrm{Y}$ and translational output $\mathrm{x}$, and three input-output control modes, and were decoupled. Based on the Jacobian determinant analysis mechanism singularity configuration, when $\theta_{R_{1}}=0^{\circ}, \theta_{R_{1}}=180^{\circ}$ and $x=\sqrt{l_{6}^{2}-\left(L_{0}-l_{5}\right)^{2}}+l_{1}$, the mechanism will have mixed singularity. Discrete element simulation and experiment illustrates that the screening efficiency of $2 \mathrm{~T} 1 \mathrm{R}$ is higher than three kinds of single DOF.

\section{References}

[1] H. Shen, H. Zhang, B. He, X. Xin, X. Huang, T. Yang, "A kind of novel parallel movement vibration sieve and its kinematic research and development," Journal of Machine Design, vol. 24, no. 6, pp. 34-36, 2007.

[2] J. Xie., X. Zhang, C. Wang, Q. Yang, "Mechanism design and kinematics simulation of three translation

ISSN: 0010-8189

(C) CONVERTER 2020

www.converter-magazine.info 
Volume 2021, No. 2

tea sieving machine," Chinese Journal of Engineering Design, vol. 19, no. 3, pp. 208-212, 2012.

[3] L.U. Qing, Z.P. Wang, "Design and Performance Simulation of 3T2R Hybrid Tea Sieving Mechanism," Forestry Machinery \& Woodworking Equipment, 2018.

[4] Y. Liu, X. Han, Z. Guo, X. Jia, "Structure and Kinematics Analysis of a Novel 3-RPR Parallel Mechanism,” China Mechanical Engineering, vol. 22, no. 19, pp. 2307-2311, 2011.

[5] Q. Dong, Y. Liu, R. Li, H. Jiang, “Analysis on Kinematics of 3-PRP Planar Parallel Mechanism," Journal of Mechanical Transmission, vol. 39,no. 01, pp. 35-38, 2015.

[6] J. Xu, Z. Xie, Z. Yang, Z. Zhao, Y. Wang, "Kinematics and dynamics analysis of a lower dof spatial parallel mechanism," Journal of Physics: Conference Series, 1820(1), 012006 (8pp), 2021.

[7] C. Fan, H. Liu, Y. Zhang, G. Yuan, "Kinematics and Singularity Analysis of a Novel 2R Fully-decoupled Parallel Manipulator," China Mechanical Engineering, vol. 21, no. 11, pp. 1301-1305, 2010.

[8] C. Tian, Y. Fang, Q.J. Ge, "Design and analysis of a partially decoupled generalized parallel mechanism for 3T1R motion," Mechanism and Machine Theory, vol. 140, pp. 211-232, 2019.

[9] H. Shen, Z. Xu, K. Xu, J. Deng, T. Yang, "Design and Kinematic Analysis of Zero Coupling and Partially Decoupled 3T1R Parallel Mechanism," Transactions of the Chinese Society for Agricultural Machinery, vol. 50, no. 04, pp. 382-392, 2019.

[10] T. Sun, S. Yang, B. Lian, "Finite and Instantaneous Screw Theory in Robotic Mechanism," Springer, Singapore, 978-981-15-1943-7, 404 pages, 2020.

[11] G. Gogu, "Mobility and spatiality of parallel robots revisited via theory of linear transformations," European Journal of Mechanics -A/Solids, vol. 24, no. 4, pp. 690-711, 2005.

[12] L. Dong, "Analysis on reconfiguration principle and coupling characteristics of generalized parallel mechanisms. Harbin: Harbin Institute of Technology," 2016.

ISSN: 0010-8189

(C) CONVERTER 2020 